\title{
ORBIT ENTROPY IN NONINVERTIBLE MAPPINGS
}

\author{
UHLAND BURKART
}

(Received 19 July 1982)

\begin{abstract}
Based on the intrinsic structure of a selfmapping $T: S \rightarrow S$ of an arbitrary set $S$, called the orbit-structure of $T$, a new entropy is defined. The idea is to use the number of preimages of an element $x$ under the iterates of $T$ to characterize the complexity of the transformation $T$ and their orbit graphs. The fundamental properties of the orbit entropy related to iteration, iterative roots and iteration semigroups are studied. For continuous (differentiable) functions of $\boldsymbol{R}^{n}$ to $\mathbf{R}^{n}$, the chaos of $\mathrm{Li}$ and Yorke is characterized by means of this entropy, mainly using the method of Straffingraphs.
\end{abstract}

1980 Mathematics subject classification (Amer. Math. Soc.): 54 H 20, 28 D 20.

\section{Introduction}

The theory of discrete dynamical systems, often also termed iteration theory, has gained much interest in the last years. Since Poincaré [27] suggested studying continuous dynamical systems by discretisation using the method of surface of sections, a number of research workers in various scientific disciplines (Lorenz [18], May [20], [21], Guckenheimer et al. [11], Beddington et al. [3], Hénon [13], Nagashima [26]) successfully used discrete models to investigate complicated behaviour of systems.

The mathematical treatment of such simple models (Myrberg [24], [25], Guckenheimer [12], Šarkovskii [29], Mira [22], Block [4], [5] and others) has shown that the iterative sequences of functions and therefore the states of systems can exhibit rather complicated behaviour.

(ㄷ) 1986 Australian Mathematical Society $0263-6115 / 86 \$ A 2.00+0.00$ 
To characterize such systems, a number of entropy-notions is used, depending upon whether the state space of a system is a measure space (entropy of measure preserving transformations, Rokhlin [28]), a metric space (Kolmogorov's $\varepsilon$ entropy, Kolmogorov, Tihomirov [16]) or a topological space (topological entropy of continuous functions, Adler, Konheim, McAndrew [1]).

Since for general systems the structure of the state spaces is not always given, a more general entropy, depending on the intrinsic structure of the transformation, is needed. Moreover, it would be advantageous to characterize systems not by an entropy but rather by an entropy function, thus taking into account local differences of complexity in the states of a system.

Interactions of intrinsic structures of transformations with structures of the underlying state spaces may then be investigated to get further insight into the behaviour of a system (Graw [10]).

\section{Definitions and preliminary results}

Let us recall the notion of orbit (for a detailed discussion see Targonski [31]).

Definition. Let $T: S \rightarrow S$ be a transformation from an arbitrary set $S$ on or into itself. Two elements $x, y \in S$ are equivalent under $T, x \sim_{T} y$, if and only if for some $n, m \in \mathbb{N}, T^{n}(x)=T^{m}(y)$, where $T^{n}$ means the $n$-th iterate of $T$, and $T^{0}:=$ id.

The equivalence classes under $\sim{ }_{T}$ are called orbits of $T$.

An orbit can be represented by a directed graph as follows. The elements of $S$ are represented as points of the plane and a point $x \in S$ is joined to a point $y \in S$ if and only if $y=T(x)$.

The orbit structure is an intrinsic structure of $T$. The complexity under iteration of a transformation $T$ is characterized by the complexity of the corresponding orbit graphs.

The idea now is to use the number of preimages of an element of $S$ to define a new entropy. It is exactly this number of preimages that gives the structures of orbits.

DEFINITION. Let $S$ be an arbitrary set. Define card: $P(S) \rightarrow N \cup\{\infty\}$ by

$$
\text { card } A:= \begin{cases}m & \text { if } A \text { has } m \text { elements, } \\ 1 & \text { if } A=\varnothing, \\ \infty & \text { otherwise, }\end{cases}
$$

where $A \in P(S)$, and $P(S)$ denotes the power set of $S$. 
Definition. Let $T: S \rightarrow S$ be a selfmapping of $S$. The function $\bar{h}_{T}: S \rightarrow \mathbb{R}_{0}^{+} \cup$ $\{\infty\}$ defined by $\bar{h}_{T}(x):=\lim \sup _{n \rightarrow \infty} \frac{1}{n} \log$ card $T^{-n}(x)$ is called the upper orbit entropy function of $T$, and the function $\underline{h}_{T}: S \rightarrow \mathbb{R}_{0}^{+} \cup\{\infty\}$ defined by $\underline{h}_{T}(x):=\liminf _{n \rightarrow \infty} \frac{1}{n} \log$ card $T^{-n}(x)$ is called the lower orbit entropy function of $T$. If $\underline{h}_{T}(x)=\bar{h}_{T}(x)$ for all $x \in A \subset S$, then $h_{T}(x):=\underline{h}_{T}(x)$ is called the orbit entropy function of $T$ on $A$.

REMARK. (1) $\Delta h_{T}(x):=\bar{h}_{T}(x)-\underline{h}_{T}(x)$ may be called the orbit entropy uncertainty function.

(2) For traditional reasons we take log to the base 2 .

(3) We write $\bar{h}(x)$ for $\bar{h}_{T}(x)$ whenever the transformation in question is clear.

Before we give some simple properties of the entropy functions, we treat an example.

ExAmple. Consider the Čebyšev polynomials $T_{n}: \mathbb{C} \rightarrow \mathbb{C}$ defined by

$$
\begin{aligned}
T_{0}(z) & :=2, \\
T_{1}(z) & :=z, \\
T_{n+2}(z) & :=z \cdot T_{n+1}(z)-T_{n}(z) .
\end{aligned}
$$

The functions $T_{n}$ have the following properties:

(1) $T_{n}$ is a polynomial of degree $n$;

(2) for all $n \in \mathbb{N}, T_{n}(\mathbb{R}) \subset \mathbb{R}, T_{n}([-2,2])=[-2,2], T_{n}(2)=2, T_{n}(-2)=(-1)^{n}$. 2 ;

(3) $T_{n} \mid \mathbb{R}, n \geqslant 2$, has $(n-1)$ different local extrema $x_{i}$ where

$$
T_{n}\left(x_{i}\right)=2 \text { or } T_{n}\left(x_{i}\right)=-2 \text {; }
$$

(4) $T_{n} \circ T_{m}=T_{n \cdot m}, n, m \in \mathbb{N}_{0}$, where "。" denotes composition of transformations.

Therefore we have, for $n \geqslant 2$,

(i) every $z \in \mathbb{C}, z \notin\{-2,2\}$ has exactly $n$ different preimages under $T_{n}$, which implies

$$
\begin{aligned}
h(z)=\bar{h}(z)=\underline{h}(z) & =\lim _{k \rightarrow \infty} \frac{1}{k} \log \operatorname{card} T_{n}^{-k}(z) \\
& =\lim _{k \rightarrow \infty} \frac{1}{k} \log n^{k} \\
& =\log n,
\end{aligned}
$$


(ii) for $z=2$ or $z=-2$, we have from (3) and (i), that $z$ has at least $n / 2-1$ different preimages. Hence

$$
\begin{aligned}
\left(\frac{n}{2}-1\right) \cdot n^{k-1} & \leqslant \operatorname{card} T_{n}^{-k}(z) \leqslant n^{k}, \text { which implies } \\
\bar{h}(z) & =\limsup _{k \rightarrow \infty} \frac{1}{k} \log \operatorname{card} T_{n}^{-k}(z) \\
& \leqslant \limsup _{k \rightarrow \infty} \frac{1}{k} \log n^{k} \\
& =\log n, \\
\underline{h}(z) & =\lim _{k \rightarrow \infty} \frac{1}{k} \log \operatorname{card} T_{n}^{-k}(z) \\
& \left.\geqslant \liminf _{k \rightarrow \infty} \frac{1}{k} \log \left[\left(\frac{n}{2}-1\right) n^{k-1}\right)\right] \\
& =\log n,
\end{aligned}
$$

whence $h(z)=\log n$.

Since furthermore for $n=1, T_{1}(z)=z$ we find that $h(z)=\lim _{k \rightarrow \infty} \frac{1}{k} \log 1=0$ $=\log 1$, and therefore we have, for all $n \in \mathbb{N}, h_{T_{n}}(z)=\log n$, for all $z \in \mathbb{C}$.

REMARK. (1) From (4) we have specially for Čebyšev polynomials that

$$
\begin{aligned}
h_{T_{n} \circ T_{m}}(z)=h_{T_{n} \cdot m}(z) & =\log n \cdot m \\
& =\log n+\log m \\
& =h_{T_{n}}(z)+h_{T_{m}}(z) .
\end{aligned}
$$

(2) If one considers $T_{n}$ on $\mathbb{R}$ rather than on $\mathbb{C}$, one easily derives

$$
\begin{array}{ll}
h_{T_{n}}(x)=\log n, & x \in[-2,2], \\
h_{T_{n}}(x)=0, & x \notin[-2,2] .
\end{array}
$$

We now give some simple properties of the entropy functions.

PROPOSITION 1. Let $T$ be a selfmapping of an arbitrary set $S$ and let every orbit of $T$ be of finite cardinality. Then $h(x)=0$ for all $x \in S$.

Proposition 2. Let $T$ be an injection of a set $S$. Then $h(x)=0$ for all $x \in S$.

Proposition 3. Let T: $S \rightarrow S$ and $U: Y \rightarrow Y$ be orbit isomorphic, i.e. there exists a bijection $H: S \rightarrow Y$ such that $H \circ T=U \circ H$. Then $\bar{h}_{T}(x)=\bar{h}_{U}(H(x))$ for all $x \in S$ and $\underline{h}_{T}(x)=\underline{h}_{U}(H(x))$ for all $x \in S$. 
The proofs of Proposition 1 to 3 are trivial.

Let us now recall the notions of splinter and cycle.

Definition. For $T: S \rightarrow S$, the set $S(x):=\left\{T^{n}(x) \mid n \in \mathbb{N} \cup\{0\}\right\}$ is called the splinter or iterative sequence of $x \in S$.

Definition. A cycle of order $k$ of a mapping $T: S \rightarrow S$ is a $k$-tuple of pairwise different elements of $S, x_{0}, \ldots, x_{k-1}$, such that $T\left(x_{i}\right)=x_{i+1}(0 \leqslant i<k-1)$ and $T\left(x_{k-1}\right)=x_{0}$.

Proposition 4. For $T: S \rightarrow S$, the entropy functions $\bar{h}$ and $\underline{h}$ are increasing on the splinters of $T$, that is $\bar{h}(x) \leqslant \bar{h}(T(x))$ and $\underline{h}(x) \leqslant \underline{h}(T(x))$.

Proof. From $T^{-n}(x) \subset T^{-(n+1)}(T(x))$ we have

$$
\begin{aligned}
\limsup _{n \rightarrow \infty} \frac{1}{n} \log \operatorname{card} T^{-n}(x) & \leqslant \limsup _{n \rightarrow \infty} \frac{1}{n} \log \operatorname{card} T^{-(n+1)}(T(x)) \\
& =\limsup _{n \rightarrow \infty} \frac{1}{n+1} \log \operatorname{card} T^{-(n+1)}(T(x)) \\
& =\limsup _{n \rightarrow \infty} \frac{1}{n} \log \operatorname{card} T^{-n}(T(x))
\end{aligned}
$$

which implies $\bar{h}(x) \leqslant \bar{h}(T(x))$.

Similarly, $\underline{h}(x) \leqslant \underline{h}(T(x))$.

COROLLARY. $\underline{h}(x)$ and $\bar{h}(x)$ are constant on the cycles of $T$.

\section{Orbit entropy and iteration}

In this section we discuss the connection between the entropy of a transformation $T$ and the entropy of the iterate $T^{k}$, the fractional iterate $T^{1 / k}$ (if it exists) and the continuous iterate $T^{t}, t \in \mathbb{R}_{0}^{+}$(if it exists).

THEOREM 1, Let $T: S \rightarrow S$ and $T^{k}, k \in \mathbb{N}$, be the $k$-th iterate of $T$.

(a) $\bar{h}_{T^{k}}(x) \leqslant k \cdot \bar{h}_{T}(x), \underline{h}_{T^{k}}(x) \leqslant k \cdot \underline{h}_{T}(x)$ for all $x \in S$.

(b) If $\bar{h}_{T}(x)=\underline{h}_{T}(x)=h_{T}(x)$, then $h_{T^{k}}(x)$ exists and $h_{T^{k}}(x)=k \cdot h_{T}(x)$ for all $x \in S$. 
Proof. (a) From $\frac{1}{n} \log$ card $\left(T^{k}\right)^{-n}(x)=\frac{1}{n} \log$ card $T^{-n k}(x)=k$ $\cdot \frac{1}{n \cdot k} \log \operatorname{card} T^{-n k}(x)$ we have

$$
\begin{aligned}
\bar{h}_{T^{k}}(x) & =\limsup _{n \rightarrow \infty} \frac{1}{n} \log \operatorname{card}\left(T^{k}\right)^{-n}(x) \\
& =k \cdot \limsup _{n \rightarrow \infty} \frac{1}{n \cdot k} \log \operatorname{card} T^{-n k}(x) \\
& \leqslant k \cdot \limsup _{n \rightarrow \infty} \frac{1}{n} \log \operatorname{card} T^{-n}(x) \\
& =k \cdot \bar{h}_{T}(x) .
\end{aligned}
$$

Similarly we get $\underline{h}_{T^{k}}(x) \leqslant k \cdot \underline{h}_{T}(x)$.

(b) From $\bar{h}_{T}(x)=\underline{h}_{T}(x)=h_{T}(x)$ we have

$$
\begin{aligned}
\limsup _{n \rightarrow \infty} \frac{1}{n} \log \operatorname{card} T^{-n}(x) & =\lim _{n \rightarrow \infty} \frac{1}{n} \log \operatorname{card} T^{-n}(x) \\
& =\lim _{n \rightarrow \infty} \frac{1}{n \cdot k} \log \operatorname{card} T^{-n k}(x)
\end{aligned}
$$

and hence

$$
\begin{aligned}
& \bar{h}_{T^{k}}(x)=k \cdot \bar{h}_{T}(x), \\
& \underline{h}_{T^{k}}(x)=k \cdot \underline{h}_{T}(x), \\
& \bar{h}_{T^{k}}(x)=k \cdot h_{T}(x)=\underline{h}_{T^{k}}(x)=h_{T^{k}}(x) .
\end{aligned}
$$

THEOREM 2. Let $T: S \rightarrow S$ and assume $G:=T^{1 / k}$, the $k$-th iterative root of $T$, exists for some $k \in \mathbb{N}$.

(a) $\bar{h}_{G}(x) \geqslant \frac{1}{k} \bar{h}_{T}(x)$ and $\underline{h}_{G}(x) \leqslant \frac{1}{k} \cdot \underline{h}_{T}(x)$ for all $x \in S$.

(b) If $h_{T}(x)$ and $h_{G}(x)$ exist for an $x \in S$, then $h_{G}(x)=\frac{1}{k} h_{T}(x)$.

(c) If $T$ is a surjection and $h_{T}(x)$ exists for an $x \in S$, then $h_{G}(x)$ exists and $h_{G}(x)=\frac{1}{k} h_{T}(x)$.

Proof. (a) From $G^{k}(x)=T(x)$ we have card $G^{-n k}(x)=\operatorname{card} T^{-n}(x)$.

Therefore

$$
\begin{aligned}
k \cdot \bar{h}_{G}(x) & =k \limsup _{n \rightarrow \infty} \frac{1}{n} \log \operatorname{card} G^{-n}(x) \\
& \geqslant k \limsup _{n \rightarrow \infty} \frac{1}{n \cdot k} \log \operatorname{card} G^{-n k}(x) \\
& =\limsup _{n \rightarrow \infty} \frac{1}{n} \log \operatorname{card} T^{-n}(x) \\
& =\bar{h}_{T}(x)
\end{aligned}
$$

and hence $\bar{h}_{G}(x) \geqslant \frac{1}{k} \bar{h}_{T}(x)$. Similarly $\underline{h}_{G}(x) \leqslant \frac{1}{k} \underline{h}_{T}(x)$. 
(b) From the assumptions we derive

$$
\begin{aligned}
k \cdot h_{G}(x) & =k \lim _{n \rightarrow \infty} \frac{1}{n} \log \operatorname{card} G^{-n}(x) \\
& =\lim _{n \rightarrow \infty} \frac{k}{n \cdot k} \log \operatorname{card} G^{-n k}(x) \\
& =\lim _{n \rightarrow \infty} \frac{1}{n} \log \operatorname{card} T^{-n}(x) \\
& =h_{T}(x) .
\end{aligned}
$$

(c) Since $T$ is a surjection, so is $G$ and therefore

$$
\begin{aligned}
\operatorname{card} T^{-n}(x) & \leqslant \operatorname{card} G^{-n k}(x) \\
& \leqslant \operatorname{card} G^{-n k-p}(x) \quad(p=1, \ldots, k) \\
& \leqslant \operatorname{card} G^{-k(n+1)}(x) \quad(n \in \mathbb{N}) .
\end{aligned}
$$

From the monotonicity of the log-function we then conclude

$$
\begin{aligned}
\frac{1}{k \cdot n+p} \log \operatorname{card} T^{-n}(x) & \leqslant \frac{1}{k \cdot n+p} \log \operatorname{card} G^{-k n}(x) \\
& \leqslant \frac{1}{k \cdot n+p} \log \operatorname{card} G^{-k \cdot n-p}(x) \\
& \leqslant \frac{1}{k \cdot n+p} \log \operatorname{card} G^{-k(n+1)}(x) \\
& =\frac{1}{k \cdot n+p} \log \operatorname{card} T^{-(n+1)}(x) \quad(n \in \mathbb{N}) .
\end{aligned}
$$

Taking the $\liminf _{n \rightarrow \infty}$ and $\lim \sup _{n \rightarrow \infty}$ of these inequalities we get

$$
\frac{1}{k} h_{T}(x) \leqslant \liminf _{n \rightarrow \infty} \frac{1}{k \cdot n+p} \log \operatorname{card} G^{-k n-p}(x), \quad p=1, \ldots, k,
$$

and hence $\frac{1}{k} h_{T}(x) \leqslant \underline{h}_{G}(x)$ and

$$
\begin{aligned}
& \limsup _{n \rightarrow \infty} \frac{1}{k \cdot n+p} \log \operatorname{card} G^{-k n-p}(x) \leqslant \frac{1}{k} \bar{h}_{T}(x), \quad p=1, \ldots, k, \\
& \frac{1}{k} h_{T}(x) \leqslant \underline{h}_{G}(x) \leqslant \bar{h}_{G}(x) \leqslant \frac{1}{k} h_{T}(x) .
\end{aligned}
$$

REmark. These results are just what one would expect: $T^{k}$ is a rougher description of a system since one looks at it at greater "time"-intervals. The entropy, interpreted as a measure of certainty of a state of the system, is therefore 
greater. $T^{1 / k}$ gives a more accurate description of a system and accordingly the entropy is smaller.

The next result is stated as a lemma since we mainly use it for the proof of the next theorem, but nevertheless it is interesting in itself.

Lemma. Let $T: S \rightarrow S$ and $U: S \rightarrow S$ be commuting surjections, that is $T \circ U(x)$ $=U \circ T(x)$ for all $x \in S$, and let $V(x):=T \circ U(x)$. Then

$$
\begin{array}{ll}
\underline{h}_{T}(x) \leqslant \underline{h}_{V}(x), & \bar{h}_{T}(x) \leqslant \bar{h}_{V}(x), \\
\bar{h}_{U}(x) \leqslant \underline{h}_{V}(x), & \bar{h}_{U}(x) \leqslant \bar{h}_{V}(x) .
\end{array}
$$

Proof. Since $U$ and $T$ commute, it follows by induction that

$$
V^{n}=(U \circ T)^{n}=U^{n} \circ T^{n}=T^{n} \circ U^{n}
$$

and therefore

$$
\operatorname{card} V^{-n}(x)=\operatorname{card} T^{-n}\left(U^{-n}(x)\right)=\operatorname{card} U^{-n}\left(T^{-n}(x)\right) .
$$

From the surjectivity we then derive

$$
\operatorname{card} U^{-n}(x) \leqslant \operatorname{card} V^{-n}(x), \quad \operatorname{card} T^{-n}(x) \leqslant \operatorname{card} V^{-n}(x) .
$$

By the monotonicity of the log-function we finish the proof by taking the $\liminf _{n \rightarrow \infty}$ and $\lim \sup _{n \rightarrow \infty}$.

THEOREM 3. Let $T: S \rightarrow S$ be a surjection and $h_{T}(x)<\infty$. Furthermore let $T$ be imbeddable into an iterative semigroup, that is there exists a family of mappings $T^{t}$ : $S \rightarrow S, t \in \mathbb{R}_{0}^{+}$, such that $T^{0}=\mathrm{id}, T^{1}=T$ and $T^{s+t}=T^{s} \circ T^{t}=T^{t} \circ T^{s}$ for all $t$, $s \in \mathbb{R}_{0}^{+}$. Then $h_{T^{\prime}}(x)$ exists for all $t \in \mathbb{R}_{0}^{+}$and $h_{T^{\prime}}(x)=t \cdot h_{T}(x)$.

Proof. One easily observes that $T^{t}$ is a surjection for all $t \in \mathbb{R}_{0}^{+}$, since $T$ is a surjection. From Theorem 1 and Theorem 2, $h_{T^{r}}(x)$ exists for all $r \in Q$, and $h_{T^{r}}(x)=r \cdot h_{T}(x)$.

Consider now a fixed $t \in \mathbb{R}_{0}^{+}$. Let $\left(r_{n}\right)$ be a monotone increasing sequence converging to $t, r_{n} \in \mathbb{Q}_{0}^{+}$. Then

$$
T^{\prime}(x)=T^{r_{n}} \circ T^{t-r_{n}}(x) .
$$

Now $T^{r_{n}}$ and $T^{t-r_{n}}$ commute by assumption, and from the lemma we have $h_{T^{r_{n}}}(x)=r_{n} \cdot h_{T}(x) \leqslant \underline{h}_{T^{\prime}}(x) \leqslant \bar{h}_{T^{\prime}}(x)$, and

$$
\lim _{n \rightarrow \infty} h_{T^{r_{n}}}(x)=\lim _{n \rightarrow \infty} r_{n} \cdot h_{T}(x)=t \cdot h_{T}(x) \leqslant \underline{h}_{T^{\prime}}(x) \leqslant \bar{h}_{T^{\prime}}(x) .
$$


Let now $\left(s_{n}\right)$ be a monotone decreasing sequence converging to $t, s_{n} \in \mathbb{Q}_{0}^{+}$. Similarly we conclude from $T^{t} \circ T^{s_{n}-t}=T^{s_{n}}$ that

$$
\lim _{n \rightarrow \infty} h_{T^{s_{n}}}(x)=\lim _{n \rightarrow \infty} s_{n} \cdot h_{T}(x)=t \cdot h_{T}(x) \geqslant \bar{h}_{T^{\prime}}(x) \geqslant \underline{h}_{T^{\prime}}(x) .
$$

Therefore $t \cdot h_{T}(x) \leqslant \underline{h}_{T^{\prime}}(x) \leqslant \bar{h}_{T^{t}}(x) \leqslant t \cdot h_{T}(x)$.

\section{Orbit entropy and chaos}

Since the paper of $\mathrm{Li}$ and Yorke [17] where they suggested the notion of "chaos" to describe the irregular behaviour of the splinters of a continuous selfmapping of $\mathbb{R}$, a number of authors have dealt with this notion and generalized it to continuous (respectively differentiable) selfmappings of $\mathbb{R}^{n}$, giving sufficient conditions for chaos to appear (Diamond [9], Kloeden [15], Marotto [19]). For the definitions of chaos and further notions that will appear throughout this section we refer to the appendix. Since chaos appears in different ways it is natural to ask whether it can be characterized quantitatively. We shall see that the lower orbit entropy function provides a possibility to do this.

First we construct for a given mapping $T: \mathbb{R}^{n} \rightarrow \mathbb{R}^{n}$ certain directed graphs, the Straffin-graphs (Straffin [30], Burkart [8]). Let $A_{i}$ and $A_{j}$ be two nonvoid subsets of $\mathbb{R}^{n}$. We say that $A_{i}$ and $A_{j}$ are related by $T$ if and only if $T\left(A_{i}\right) \supset A_{j}$ or $T\left(A_{j}\right) \supset A_{i}$. Let now $A_{1}, \ldots, A_{k} \subset \mathbb{R}^{n}$ be nonvoid sets, where each set $A_{i}$ is related to at least one other set $A_{j}, 1 \leqslant i \leqslant k, 1 \leqslant j \leqslant k$. We then consider the sets $A_{1}, \ldots, A_{k}$ as vertices of a graph and draw a directed edge from $A_{i}$ to $A_{j}$ if and only if $T\left(A_{i}\right) \supset A_{j}$. If $A_{j}$ is covered by $T\left(A_{i}\right)$ more than once, that is if there exist disjoint subsets $A_{i_{m}}, 1 \leqslant m \leqslant p$, of $A_{i}$ with $T\left(A_{i_{m}}\right) \supset A_{j}$, we draw $p$ edges from $A_{i}$ to $A_{j}$. Note that the number $p$ may not be exactly determined. The resulting graph we call a Straffin-graph ( $S$-graph) of $T$ related to $A_{1}, \ldots, A_{k}$.

A $q$-cycle in a S-graph is a walk from a vertex $A_{i}$ along the edges of the graph back to $A_{i}$ in $q$ steps.

TheOREM 4. Let $T: \mathbb{R}^{n} \rightarrow \mathbb{R}^{n}$ be continuous with a q-cycle $A_{1}, \ldots, A_{q}$ in a $S$-graph. Let furthermore $m_{i}$ denote the number of edges from $A_{i}$ to $A_{i+1}, i=$ $1, \ldots, q-1$, and $m_{q}$ the number of edges from $A_{q}$ to $A_{1}$. If $\sum_{i=1}^{n} m_{i}>q$, then $\underline{h}_{T}(x)>0$ for all $x \in A_{i}$, and $\underline{h}_{T}(x) \geqslant \frac{1}{q} \sum_{i=1}^{q} \log m_{i}$.

Proof. Let $x \in A_{i}$. Since $T \mid \cup_{i=1}^{q} A_{i}$ is surjective and $\underline{h}$ is increasing on the splinter of $x$, and furthermore $\sum_{i=1}^{q} m_{i}>q$, we can assume without loss of generality that $x$ is not a periodic point of $T$ (otherwise we consider a nonperiodic 
predecessor of $x$ ). Now

$$
\operatorname{card} T^{-q}(x) \geqslant \prod_{i=1}^{q} m_{i}
$$

by the disjointness of the $A_{i_{k}}, 1 \leqslant k \leqslant m_{i}$, and therefore

$$
\operatorname{card} T^{-q \cdot p}(x) \geqslant \prod_{i=1}^{q} m_{i}^{p}
$$

Since $T \mid \bigcup_{i=1}^{q} A_{i}$ is surjective we get $\log \operatorname{card} T^{-r}(x) \geqslant \log \prod_{i=1}^{q} m_{i}^{s}$, where $s \in \mathbb{N}$ is maximal such that $q \cdot s \leqslant r$. Hence

$$
\frac{q}{r} \log \operatorname{card} T^{-r}(x) \geqslant \frac{q}{r} \log \prod_{i=1}^{q} m_{i}^{s}=\frac{q \cdot s}{r} \log \prod_{i=1}^{q} m_{i} .
$$

Let now $\varepsilon>0$ be arbitrary. Then for a certain $r_{0}(\varepsilon) \in \mathbb{N}$ we have

$$
\frac{q \cdot s}{r}>\frac{1}{(1+\varepsilon)}
$$

for all $r>r_{0}$, where $s$ is maximal such that $q \cdot s<r$. Hence

$$
\begin{aligned}
q \cdot \liminf _{r \rightarrow \infty} \frac{1}{r} \log \operatorname{card} T^{-r}(x) & \geqslant \frac{1}{(1+\varepsilon)} \log \prod_{i=1}^{q} m_{i} \\
& \Rightarrow \underline{h}(x) \geqslant \frac{1}{q(1+\varepsilon)} \log \prod_{i=1}^{q} m_{i} \\
& \Rightarrow \underline{h}(x) \geqslant \frac{1}{q} \sum_{i=1}^{q} \log m_{i} .
\end{aligned}
$$

Since $\sum_{i=1}^{q} m_{i}>q$, there exists a least one $m_{i}$ with $m_{i} \geqslant 2$. Therefore $\underline{h}(x)>0$.

TheORem 5. Let $T: \mathbb{R}^{n} \rightarrow \mathbb{R}^{n}$ be continuous and let there exist an $S$-graph of $T$ that contains at least $t$ wo cycles $A_{1}, \ldots, A_{q}$ and $B_{1}, \ldots, B_{p}$ with $A_{i}=B_{j}$ for a pair $i, j$ and at least one $B_{u}$ that is disjoint from all $A_{i}, i=1, \ldots, q$. Then $\underline{h}(x)>0$ for all $x \in \bigcup_{i=1}^{q} A_{i} \cup \bigcup_{j=1}^{p} B_{j}$, and $\underline{h}(x) \geqslant 1 / q \cdot p$.

ProOF. Without loss of generality we may again assume that an arbitrary point $x \in A_{i}$ is nonperiodic (otherwise we consider a predecessor of $x$ ). From the assumptions of the theorem it follows that $x$ has at least two different predecessors under $T^{p \cdot q}$. From the surjectivity of $T \mid \cup_{i=1}^{q} A_{i} \cup \bigcup_{j=1}^{p} B_{j}$ we conclude that card $T^{-m}(x) \geqslant 2^{k}$, where $k \in \mathbb{N}$ is maximal such that $p \cdot q \cdot k \leqslant m$. By a reasoning similar to the proof of Theorem 4 we now find that

$$
p \cdot q \liminf _{m \rightarrow \infty} \frac{1}{m} \log \text { card } T^{-m}(x) \geqslant \frac{1}{(1+\varepsilon)} \log 2 \text { for all } \varepsilon>0,
$$

and therefore $\underline{h}(x) \geqslant \frac{1}{p \cdot q}$. From the monotonicity of $\underline{h}$ on the splinters of $T$ it follows that this result holds for all $x \in \bigcup_{i=1}^{q} A_{i} \cup \bigcup_{j=1}^{p} B_{j}$. 
REMARK. If one knows the structure of the S-graph more precisely one can derive better results. For example, the famous 3-periodic continuous functions of an interval of $I \subset \mathbb{R}$ that are studied by Li-Yorke [17] allow for S-graphs that consist of a two-cycle and a loop (Straffin [30]). Therefore $\underline{h}(x) \geqslant 1 / 2$ from Theorem 5. From the special structure of this S-graph it is, however, easy to see that card $T^{-m}(x) \geqslant v_{m}$ for $x \in A_{1} \cup A_{2}$, where $v_{m}=v_{m-1}+v_{m-2}, v_{1}=1, v_{2}=2$.

We therefore have the Fibonacci numbers for an estimation:

$$
\begin{aligned}
\underline{h}_{T}(x) & \geqslant \lim _{m \rightarrow \infty} \frac{1}{m} \log \left[\left(\frac{1+\sqrt{5}}{2}\right)^{m}+\left(\frac{1-\sqrt{m}}{2}\right)^{m}\right] \\
& =\log \frac{1+\sqrt{5}}{2}=0.69424 \cdots .
\end{aligned}
$$

Corollary. Let $T: \mathbb{R} \rightarrow \mathbb{R}$ be continuous and chaotic. Then there exists a nonvoid set $A \subset \mathbb{R}$ with $\underline{h}_{T}(x)>0$ for all $x \in A$.

Proof. Since $T$ is chaotic, there exists a point of period not a power of two. Therefore an S-graph fulfilling the assumptions of Theorem 5 can be constructed (Burkart [7]).

We now study the case of chaos in $\mathbb{R}^{n}$, that is, the sufficient conditions for chaos given by Kloeden [15] and Marotto [19].

TheOREM 6. Let $T: \mathbb{R}^{n} \rightarrow \mathbb{R}^{n}$ be continuous and let $A \subset \mathbb{R}^{n}$ and $B \subset \mathbb{R}^{n}$ be nonvoid compact sets such that there exist $n_{1}, n_{2} \in \mathbb{N}$ for which

(a) $A \subset T(A)$,

(b) $B \subset A$,

(c) $T^{n_{1}}(B) \cap A=\varnothing$,

(d) $T^{n_{1}+n_{2}}(B) \supset A$,

Then $\underline{h}(x) \geqslant 1 /\left(n_{1}+n_{2}\right)$ for all $x \in A$.

Proof. From (d) and (b) we have $T^{n_{1}+n_{2}}(B) \supset A \supset B$. Since $B$ is compact there exists a nonvoid compact set $D \subset B \subset A$ such that $T^{n_{1}+n_{2}}(D)=A$ by the continuity of $T^{n_{1}+n_{2}}$. From (c) we then conclude that

$$
T^{n_{1}}(D) \cap A=\varnothing .
$$

Consider now the S-graph of $T$ that is given by the sets $A, T(D), T^{2}(D), \ldots$, $T^{n_{1}+n_{2}}(D)=A$. The graph consists of a cycle of length $n_{1}+n_{2}$ and a loop in $A$ by (a). From (1) we conclude that the conditions of Theorem 5 are fulfilled, and therefore $\underline{h}(x) \geqslant 1 /\left(n_{1}+n_{2}\right)$ for all $x \in A \cup \bigcup_{i=1}^{n_{1}+n_{2}} T^{i}(D)$. 
REMARK. The chaos-conditions of Kloeden are somewhat stronger than the conditions (a)-(d) (see appendix). It is therefore reasonable to propose that the class of transformations $T: \mathbb{R}^{n} \rightarrow \mathbb{R}^{n}$ with positive entropy functions is greater than the class of chaotic functions. A proof of this assumption unfortunately is still missing, with the exception of the case $n=1$. For this case an example has been given by Burkart [7].

TheOREM 7. Let $T: \mathbb{R}^{n} \rightarrow \mathbb{R}^{n}$ be differentiable and let there exist a snap-back repeller for $T$. Then there exists a nonvoid, compact set $A \subset \mathbb{R}^{n}$ with $h(x)>0$ for all $x \in A$.

Proof. Let $z$ be the snap-back repeller. This means (Marotto [19]) that there exist $s>0, y \in U_{s}(z)$ and $v \in \mathbb{N}$ such that $T^{k}(y) \notin U_{s}(z)$ for $1 \leqslant k<v$, and $T^{v}(y)=z$. Moreover, $T$ is expansive on $U_{s}(z)$. Since $y \in U_{s}(z)$, there exists an

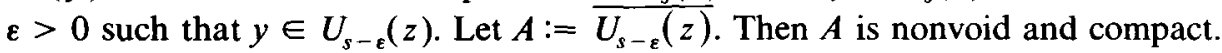
Now we take $m_{1}, 1 \leqslant m_{1}<v$, such that $T^{m_{1}}(y) \notin U_{s}(z)$, whence $T^{m_{1}}(y) \notin A$. Since $T^{m_{1}}$ is continuous, we can choose $U_{\delta}(y)$ such that for $B:=U_{\delta}(y)$ we have that $B$ is nonvoid, compact, $B \subset A$ and $T^{m_{1}}(B) \cap A=\varnothing$. Now $T^{v}(y)=z$, whence $T^{m}(y)=z$ for $m \geqslant v$, since $z$ is a fixed point of $T$. Note that $T^{m}$ is expansive on $U_{s}(z)$, since $T$ is expansive, and therefore we can choose $m_{2} \in \mathbb{N}$, $T^{m_{2}}(B) \supset A$. Finally, we have $T(A) \supset A$ from the expansitivity of $T$ on $A \subset$ $U_{s}(z)$. Hence the conditions of Theorem 5 are fulfilled.

\section{Discussion}

The proposed notion of orbit entropy functions has been shown to make sense since these functions fulfill the conditions that one likes to have for an entropy. Furthermore this type of entropy can be used to estimate quantitatively the amount of chaos that appears in the description of some systems. If, for example, one considers the transformation $T:[0,1] \rightarrow[0,1], T(x):=r \cdot x(1-x)$ that is often considered as a model for biological populations depending on the parameter $r$, one can easily discuss the S-graphs of $T$ that are changing their form under variation of $r$. With increasing $r(0<r<4)$, more and more complicated splinters appear (Guckenheimer et al. [11]), entering into a chaos situation for a critical $r$. The structure of the chaos changes by further increase of $r$ and results in an increase of the orbit entropy functions (Burkart [7]). The same behaviour can be observed in higher dimensional models (Guckenheimer et al. [11], Marotto [19]), and the entropy functions behave accordingly. It has to be stated, however, that up to now we have only obtained estimations for the entropy, and there is a 
lot of work to be done: for example to find classes of transformations for which the entropy functions exist and can be derived. Unfortunately not very much is known about the orbit structures of functions (see Targonski [31]).

Let us conclude with some remarks on the connection between topological entropy and orbit entropy. Clearly the notions are different since topological entropy makes sense for homeomorphisms, whereas our entropy is zero for bijections. But let us consider the class $H$ of piecewise strictly monotonic continuous selfmappings of an interval $I \subset \mathbb{R}$. For $T \in H$, let $s_{T}$ denote the topological entropy and let $c_{n}$ be the number of extrema of $T^{n}$ in $I$. Then $s_{T}=\lim _{n \rightarrow \infty} \frac{1}{n} \log c_{n}<\infty$, as Misiurewicz and Szlenk [23] have proven. On the other hand we clearly have card $T^{-n}(x) \leqslant c_{n}$ for all $x \in I$ and therefore $\underline{h}_{T}(x)$ $\leqslant \bar{h}_{T}(x) \leqslant s_{T}$ for all $x \in I$. If, moreover, $T$ maps every interval where $T$ is either strictly increasing or strictly decreasing onto the whole of $I$, we find $\underline{h}_{T}(x)=\bar{h}_{T}(x)=s_{T}$ for all $x \in I$. As a consequence we get an easy proof of a theorem of Adler and McAndrew [2] that states that $s_{T_{n}}=\log n$, where the $T_{n}$ are the Čebyšev polynomials on [-2,2] (see our example).

The main advantage of our entropy, at least in our opinion, seems to be that we treat entropy functions.

Kloeden [14] has shown that one can construct functions that are chaotic on an arbitrarily small interval and show absolutely regular behaviour elsewhere, even perhaps the identity outside these small intervals. Now Bowen and Franks [6] prove that these functions have positive topological entropy, depending on the appearance of periodic points (see definition of chaoticity in the appendix). Therefore topological entropy only indicates that there is chaos but gives no measure at all on how "serious" this has to be taken. An entropy function clearly overcomes this disadvantage since it states at which "places" chaos actually appears.

The connection between our entropy and topological entropy in other classes than $H$, as well as the connection to other mentioned entropies, is unknown and may initiate further work.

\section{Appendix}

In this appendix we give, for the convenience of the reader, a few definitions and results related to "chaos" that are directly connected to our theorems.

Definition. A mapping $T: S \rightarrow S$ has a $k$-periodic point $x \in S$ if the points $x$, $T(x), \ldots, T^{k-1}(x)$ are a $k$-cycle under $T$. 
Definition (Li-Yorke [17], Kloeden [15]). A continuous mapping $T: \mathbb{R}^{n} \rightarrow \mathbb{R}^{n}$ is called chaotic if

(1) there exists an $N_{0} \in \mathbb{N}$ such that $T$ has periodic points of all periods $p \geqslant N_{0}, p \in \mathbb{N}$

(2) there exists an uncountable set $S \subset \mathbb{R}^{n}$ containing no periodic points such that
(a) $T(S) \subset S$,
(b) for all $x, y \in S, x \neq y$,
( $\alpha) \lim \sup _{n \rightarrow \infty}\left\|T^{n}(x)-T^{n}(y)\right\|>0$,
( $\beta) \liminf _{n \rightarrow \infty}\left\|T^{n}(x)-T^{n}(y)\right\|=0$,
(c) for all $x \in S$ and all periodic points $q \in \mathbb{R}^{n}$,

$$
\limsup _{n \rightarrow \infty}\left\|T^{n}(x)-T^{n}(q)\right\|>0 .
$$

Definition. A mapping $T: \mathbb{R}^{n} \rightarrow \mathbb{R}^{n}$ is expanding on a set $A \subset \mathbb{R}^{n}$ if there exists a constant $\lambda>1$ such that $\lambda\|x-y\| \leqslant\|T(x)-T(y)\|$ for all $x, y \in A$.

DEFINITION. An $m$-ball is a closed ball of finite radius in $\mathbb{R}^{m}$.

Definition (Marotto [19]). Let $T: \mathbb{R}^{n} \rightarrow \mathbb{R}^{n}$ with $T(z)=z$ for a $z \in \mathbb{R}^{n}$. Then $z$ is an expansive fixed point of $T$ if there exists a neighborhood $U_{r}(z)$ such that $T$ is differentiable in $U_{r}(z)$, and all eigenvalues of $D T(x)$ exceed 1 in absolute value for all $x \in U_{r}(z)$.

Definition (Marotto [19]). A point $z \in \mathbb{R}^{n}$ is called a snap-back repeller of a mapping $T: \mathbb{R}^{n} \rightarrow \mathbb{R}^{n}$ if

(a) $z$ is an expansive fixed point of $T$ in a neighborhood $U_{r}(z)$,

(b) there exists $y \in U_{r}(z), y \neq z$, such that $T^{m}(y)=z$ for an $m \in \mathbb{N}$, and $\operatorname{det}\left(D T^{m}(y)\right) \neq 0$.

THEOREM (Li-Yorke [17]). Let $T: \mathbb{R} \rightarrow \mathbb{R}$ be continuous and have a 3-periodic point. Then $T$ is chaotic.

THEOREM (Kloeden [15]). Let $T: \mathbb{R}^{n} \rightarrow \mathbb{R}^{n}$ be a continuous mapping and suppose that there exist nonempty compact sets $A$ and $B$ and integers $1 \leqslant m \leqslant n$ and $n_{1}$, $n_{2} \geqslant 1$ such that

(1) $A$ is homeomorphic to an $m$-ball,

(2) $T(A) \supset A$,

(3) $T$ is expanding on $A$,

(4) $B \subset A$, 
(5) $T^{n_{1}}(B) \cap A=\varnothing$,

(6) $T^{n_{1}+n_{2}}(B) \supset A$,

(7) $T^{n_{1}+n_{2}}$ is one-to-one on $B$.

Then $A$ is chaotic.

THEOREM (Marotto [19]). If $T: \mathbb{R}^{n} \rightarrow \mathbb{R}^{n}$ has a snap-back repeller, then $T$ is chaotic.

\section{References}

[1] R. L. Adler, A. G. Konheim and M. H. McAndrew, 'Topological entropy', Trans. Amer. Math. Soc. 114 (1965), 309-319.

[2] R. L. Adler and M. H. McAndrew, 'The entropy of Chebyshev polynomials', Trans. Amer. Math. Soc. 121 (1966), 236-241.

[3] J. Beddington, C. Free and J. Lawton, 'Dynamic complexity in predator-prey models framed in difference equations', Nature 255 (1975), 58-60.

[4] L. Block, 'Mappings of the interval with finitely many periodic points have zero entropy', Proc. Amer. Math. Soc. 67 (1977), 357-361.

[5] L. Block, 'Simple periodic orbits of mappings of the interval', Trans. Amer. Math. Soc. 254 (1979), 391-398.

[6] R. Bowen and J. Franks, 'The periodic points of maps of the disk and the interval', Topology 15 (1976), 337-342.

[7] U. Burkart, Zur Charakterisierung diskreter dynamischer Systeme (Inaugural dissertation, Universität Marburg, 1981).

[8] U. Burkart, 'Interval mapping graphs and periodic points of continuous functions', J. Combinatorial Theory Ser. B 32 (1982), 57-68.

[9] P. Diamond, 'Chaotic behaviour of systems of difference equations', Internat. J. Systems Sci. 8 (1976), 953-956.

[10] R. Graw, Über die Orbitstruktur stetiger Abbildungen (Inaugural dissertation, Universität Marburg, 1981).

[11] J. Guckenheimer, G. F. Oster and A. Ipaktchi, 'The dynamics of density dependent population models', J. Math. Biol. 4 (1977), 101-147.

[12] J. Guckenheimer, 'The bifurcation of quadratic functions', Ann. New York Acad. Sci. 316 (1979), 78-85.

[13] M. Hénon, 'A two-dimensional mapping with a strange attractor', Comm. Math. Phys. 50 (1976), 69-77.

[14] P. E. Kloeden, 'Chaotic difference equations are dense', Bull. Austral. Math. Soc. 15 (1976), 371-379.

[15] P. E. Kloeden, 'Chaotic difference equations in $\mathbb{R}^{n}$, J. Austral. Math. Soc. (Ser. A) 31 (1981), 217-225.

[16] A. N. Kolmogorov and V. M. Tihomirov, ' $\varepsilon$-entropy and $\varepsilon$-capacity of sets in functional spaces', Trans. Amer. Math. Soc. 17 (1961), 277-364.

[17] T. Y. Li and J. A. Yorke, 'Period three implies chaos', Amer. Math. Monthly 82 (1975), 985-992.

[18] E. Lorenz, 'Deterministic nonperiodic flow', J. Atmospheric Sci. 20 (1963), 130-141.

[19] F. R. Marotto, 'Snap-back repellers imply chaos in $\mathbf{R}^{n}$ ', J. Math. Anal. Appl. 63 (1978), 199-223. 
[20] R. M. May, 'Biological populations with nonoverlapping generations: stable points, stable cycles and chaos', Science 186 (1974), 645-647.

[21] R. M. May, 'Bifurcations and dynamic complexity in ecological systems', Ann. New York Acad. Sci. 316 (1979), 517-529.

[22] C. Mira, 'Systémes a dynamique complexe et bifurcations de type "boites emboitées", cas des récurrences d'ordre 1 determinées par une fonction a un seul extrémum', Partie 1 et 2, R.A.I.R.O. Automat. 12 (1978), 63-94, 171-190.

[23] M. Misiurewicz and W. Szlenk, 'Entropy of piecewise monotone mappings', Studia Math. 67 (1980), 45-63.

[24] P. J. Myrberg, 'Iteration der reellen Polynome zweiten Grades I', Ann. Acad. Sci. Fenn. Ser. A I Math. 256 (1958), 1-10.

[25] P. J. Myrberg, 'Iteration der reellen Polynome zweiten Grades II', Ann. Acad. Sci. Fenn. Ser. A I Math. 268 (1959), 1-13.

[26] H. Nagashima, 'Chaotic states in the Belousov-Zhabotinsky reaction', J. Phys. Soc. Japan 49 (1980), 2427-2428.

[27] H. Poincaré, Les méthodes nouvelles de la méchanique céleste (Vol. 3, Gauthier-Villars, Paris, 1892).

[28] V. A. Rokhlin, 'Lectures on the entropy theory of transformations with invariant measures', Russian Math. Surveys 22 (1967), 1-52.

[29] A. N. Šarkovskii, 'Coexistence of cycles of a continuous map of the line into itself' (Russian), Ukrain. Mat. ̌̌. 16 (1964), 61-71.

[30] Ph. D. Straffin, Jr., 'Periodic points of continuous functions', Math. Mag. 51 (1978), 99-105.

[31] G. Targonski, Topics in iteration theory (Studia Mathematica 6, Vandenhoeck and Ruprecht, Göttingen, Germany, 1981).

\section{Fachbereich Mathematik}

Universität Marburg

Lahnberge

D-3550 Marburg

Federal Republic of Germany 